\title{
Morbidities Related to Obesity- A Short Review of Literature
}

\author{
Lata Kanyal Butola ${ }^{1}$, Deepika Kanyal², Archana Dhok ${ }^{3}$ \\ ${ }^{1,3}$ Department of Biochemistry, Jawaharlal Nehru Medical College, Wardha, Maharashtra, India. ${ }^{2}$ Department of \\ Hospital Administration, Jawaharlal Nehru Medical College, Wardha, Maharashtra, India.
}

\section{ABSTRACT}

Obesity is a significant risk factor for cardiovascular disorders, metabolic disorders, osteoarthritis, multiple cancers, serious mental illness, hypertension, dyslipidemia, diabetes mellitus and coronary artery disease. Obesity has been reported to promote oxidative stress, as obesity stimulates the penetration and activation of macrophages and monocytes into adipose tissue which, in turn, releases elevated levels of pro-inflammatory adipokines, such as tumour necrosis factor-alpha ( TNFa) and interleukin-6 (IL-6). Insulin resistance is associated with both type 2 diabetes and obesity. Alteration of lung mechanics in obese patients, including decreased lung capacities, decreased compliance, irregular breathing and perfusion and gas exchange, and respiratory muscle inefficiency, can predispose to increased risk and severity of respiratory infections. Obesity and atherosclerosis pathogenesis have many popular causes. In all cases, lipids, oxidized LDL particles and free fatty acids trigger the inflammatory response and induce the disease. In this review article, we have focused on different morbidities related to obesity.

\section{KEYWORDS}

Obesity, Diabetes Mellitus, Hypertension, Oxidative Stress, Osteoarthritis.
Corresponding Author: Dr. Lata Kanyal Butola, Department of Biochemistry, Jawaharlal Nehru Medical College, Wardha, Maharashtra, India.

E-mail: kanyallata1010@gmail.com

DOI: $10.14260 / j e m d s / 2021 / 753$

How to Cite This Article:

Butola LK, Kanyal D, Dhok A. Morbidities related to obesity- a short review of literature. J Evolution Med Dent Sci 2021;10(43):3720-3725, $10.14260 / \mathrm{jemds} / 2021 / 753$

Submission 28-10-2020,

Peer Review 17-09-2021,

Acceptance 25-09-2021,

Published 25-10-2021.

Copyright (c) 2021 Lata Kanyal Butola et al. This is an open access article distributed under Creative Commons Attribution License [Attribution 4.0 International (CC BY 4.0)] 


\section{BACKGROUND}

The prevalence of obesity has risen dramatically in India over the last few decades. About one-third of the adult population in urban India is currently overweight or obese. As a result, the number of people with hypertension and T2DM will rise exponentially. ${ }^{1}$ Apart from contributing to T2DM and hypertension, obesity is a significant risk factor for cardiovascular disorders, metabolic disorders, osteoarthritis, multiple cancers and serious mental illness. ${ }^{2}$ Obesity in adults is well known to increase the risk of hypertension, dyslipidemia, diabetes mellitus, coronary artery disease. It is considered a non-communicable disorder that has a major effect on the quality of life (QOL) and leads to different disease conditions and disabilities in the patient's life. ${ }^{3}$

\section{MORBIDITIES RELATED TO OBESITY}

\section{Impaired Glucose Tolerance and Diabetes Mellitus}

WHO has predicted that by 2030 T2DM may affect up to 79.4 million individuals in India. ${ }^{4}$ T2DM was earlier referred to as non-insulin-dependent diabetes or adult-onset diabetes, which accounted for $90-95 \%$ of all diabetes. Diabetes mellitus is a group of metabolic disorders characterized by insulin resistance initially, impaired insulin secretion, insulin deficiency, increased glucose production and decreased glucose utilization and the complications arising from this disease is the major cause of death worldwide. The cells of the body cannot metabolize carbohydrate due to relative or complete lack of insulin and body breaks its protein, fat, and glycogen resulting in hyperglycemia. ${ }^{5,6}$ Insulin resistance is associated with both type 2 diabetes and obesity. Most obese individuals do not develop hyperglycaemia despite being insulin resistant. Pancreatic $\beta$-cells in the islet of Langerhans release sufficient amounts of insulin to overcome insulin reductions under normal circumstances, thus maintaining normal glucose tolerance. 7 Throughout the normal history of type 2 diabetes, endothelial dysfunction is characterized by obesity/insulin resistance in diabetes and pre-diabetes disorders (including those with impaired glucose tolerance and/or decreased fasting glucose). To develop insulin resistance and obesity, thus triggering type 2 diabetes, $\beta$-cells may not be able to completely compensate for reduced insulin sensitivity. "Non-esterified fatty acids (NEFAs) that are excreted from adipose tissue in obese people may contribute to the hypothesis that insulin resistance and $\beta$-cell dysfunction are most likely associated. ${ }^{8}$ Insulin sensitivity fluctuation happens during the normal life cycle. For example, insulin tolerance is observed during puberty, breastfeeding and ageing. ${ }^{9}$ In comparison, dietary variations, such as elevated ingestion of carbohydrates and greater physical exercise, are linked with increases in insulin sensitivity. ${ }^{10}$ Obesity is known to be the most significant factor in the production of metabolic disorders. Adipose tissue regulates metabolism by secreting hormones, glycerol, and other compounds, including leptin, cytokines, adiponectin, and proinflammatory compounds, and by releasing NEFAs. The secretion of these compounds would be improved in obese persons. The insulin sensitivity is determined by another important aspect, the distribution of body fat. Insulin tolerance is correlated with body mass index at some amount of weight gain. Insulin exposure also varies entirely in lean people due to variations in body fat distribution. Individuals whose fat distribution is more distant have more insulin sensitivity than individuals whose fat distribution is more central (i.e., in the abdominal and chest area). Despite their fragility, $\beta$-cells play a crucial role in the regulation of insulin release. The amount of insulin produced by $\beta$-cells fluctuates and varies depending on the size, type and route of stimulation administration. Therefore, $\beta$-cells play a very important role in ensuring that blood glucose concentrations are steady in healthy participants within a relatively normal physiological range. Obesity reduces the sensitivity of insulin as well as the regulation of $\beta$-cell function. ${ }^{11}$

\section{Hypertension}

Obesity raises the risk of developing hypertension. Obesity is a significant risk factor for critical HTN. Obesity-related HTN can arise by several mechanisms: insulin resistance, adipokine alterations, inappropriate sympathetic nervous activity and renin-angiotensin-aldosterone system activation, physiological and functional defects in the kidneys, cardiac and vascular changes, and immune maladaptation. Uric acid and incretin of dipeptidyl peptidase 4 activity alteration also contribute to the development of HTN in the context of obesity. ${ }^{12}$

\section{Oxidative Stress}

Obesity has been reported to promote oxidative stress, as obesity stimulates the penetration and activation of macrophages and monocytes into adipose tissue ${ }^{13}$ which, in turn, releases elevated levels of pro-inflammatory adipokines, such as tumour necrosis factor-alpha ( TNF-a) and interleukin-6 (IL-6). These inflammatory markers induce the production of reactive oxygen species (ROS), resulting in its formation. There are claims that oxidative stress can induce hypertension, as increased ROS can decrease NO levels by converting NO to more NO-reactive species. Reduced NO contributes to endothelial dysfunction and may inhibit endothelial vascular relaxation, thereby increasing vascular contractile activity, which may cause high blood pressure. ${ }^{14}$ Oxidative stress has been associated with lipid, protein, and DNA damage. Thiobarbituric acid-reactive substances (TBARS) are commonly formed after cell membrane injury, while 8-hydroxy-2-deoxyguanosine (8-OHdG) is commonly formed as a result of DNA degradation due to oxidative stress. ${ }^{15}$

\section{Pulmonary Abnormalities}

Alteration of lung mechanics in obese patients, including decreased lung capacities, decreased compliance, irregular breathing and perfusion and gas exchange, and respiratory muscle inefficiency, can predispose to increased risk and severity of respiratory infections. ${ }^{16}$ Overweight and obese individuals are more likely to have respiratory symptoms than average BMI individuals. Respiratory muscle control has been found to deteriorate in obesity, a trend close to that seen in chronic respiratory conditions such as COPD. This can 
also be linked to a decrease in the total fat-free mass (FFM, i.e. muscle mass). This deficiency may lead to the increased oxygen demand needed for breathing and may increase the feeling of breathlessness in obese patients. In addition, tests have found that weight loss helps to recover the function of the respiratory muscle. As a result, the increased mechanical workload of obesity can overburden the respiratory muscles by mixing increased breathing work with an obvious decrease in respiratory muscle performance. ${ }^{17}$

\section{COPD and Obesity}

COPD is marked by progressive and largely persistent airflow restriction which exists mainly in smokers. ${ }^{18}$ COPD with obesity has a dynamic interplay of characteristics that appears to compound each disease. These are linked with impairment of lung function, hypoxia, and low-grade systemic inflammation, which predisposes to elevated medical morbidity and mortality.

\section{Obesity and Anaesthesia}

By an ever-increasing requirement for surgical procedures involving anaesthesia combined with the growing global overweight population, it is not shocking that obesity has become such a common and significant consideration in the routine anaesthetic evaluation. Obesity and its underlying conditions provide an anaesthetist with various challenges involving meticulous preparation and knowledge of certain potential risks. In specific, obstructive sleep apnoea (OSA) and obesity hypoventilation syndrome (OHS) should often be considered in obese patients undertaking surgery assessments". ${ }^{19}$ Obesity can lead to complications at all stages of anaesthesia as it makes intubation and breathing difficult. Anatomical landmarks are also less simple, with regular problems in maintaining vascular access and catheterization. Growing weight makes the location of the ambulation challenging and suitable for radiographic imaging. The pharmacokinetics of medications is sometimes complicated owing to an excessive quantity of adipose tissue. Obese surgical patients face higher anaesthetic complications and frequently need longer hospital stays with increased health care costs. They are more vulnerable to postoperative cardiac and pulmonary complications, including pneumonia (associated with $30 \%-46 \%$ mortality) and thromboembolic disease. ${ }^{20}$

\section{Obesity and osteoarthritis}

Obesity is characterized by a low-grade inflammatory state, leading to its effects on multiple organ systems. ${ }^{21}$ There are currently no known exact metabolic mechanisms by which obesity contributes to joint structural injury, but it is thought to include aberrant adipokine production with upstream and downstream effects leading to the degradation and remodelling of joint tissue. ${ }^{22}$ Adipokines have effects on the joint tissue, including cartilage, synovium and bone. Leptin and adiponectin are the most abundantly developed adipokines and their receptors are expressed on the surface of chondrocytes, synoviocytes and subchondral osteoblasts. Leptin has been shown to increase the amounts of degrading enzymes, such as matrix metalloproteinases (MMPs) and nitric oxide, and the development of pro-inflammatory cytokines. ${ }^{23}$ Adipokines amounts in people with obesity may be especially significant, as obesity may create a metabolic condition in which chondrocytes cannot respond to these challenges. For example, leptin response habits of obese $\mathrm{OA}$ patients have been seen to vary from an average or overweight patient. Less is known about the role of adiponectin in joint disease, with pro-inflammatory and antiinflammatory properties reported ${ }^{21}$ relative to its systemic anti-inflammatory effects. Leptin and adiponectin levels are slightly higher in people with OA relative to controls. ${ }^{24}$

Obesity contributes to an improvement in the loading of the weight-bearing joint, which may be the most significant mechanical contribution. Knee adduction time can be a significant mechanical variable associated with the development of the OA knee.25 People with obesity have greater knee adduction moments due to elevated body fat, and participate in countervailing gait habits such as slower walking pace and raised toe-out angle. ${ }^{26}$ There is proof that in obese persons, joint cartilage might not be able to respond to higher absolute knee adduction moments during gait relative to average weight individuals ${ }^{27}$ Increased joint loading by average weight persons have not reliably been shown to be correlated with OA: for example, a longitudinal study of elderly ( $>60 \mathrm{yr}$ ) runners and non-runners has found that the existence of radiographic hip $\mathrm{OA}$ and the development of radiographic knee $\mathrm{OA}$ are comparable in both categories. It is important to remember that muscle and muscle forces have a significant effect on joint loading by the act of regulating how the load is transmitted in the joint, as well as on the stability of the joint. Decreasing muscle forces acting on joints or misaligned joints, such as quadriceps weakness, may lead to insufficient absorption of knee forces which may result in increased dynamic loads on the articular cartilage resulting in progressive degeneration. Thus, muscle fatigue, especially quadriceps, has been established as a significant risk factor in some studies. ${ }^{26}$ The importance of body structure and the intimate relationship between muscle loss and fat gain has been suggested as an important mechanism for the development of osteoarthritis, notably by Roubenoff et al. ${ }^{27}$

\section{Obesity and Atherosclerosis}

Obesity and atherosclerosis pathogenesis have many popular causes. In all cases, lipids, oxidized LDL particles and free fatty acids trigger the inflammatory response and induce the disease. Inflammation is responsible for all steps towards atherosclerosis, from early endothelial dysfunction to complication-causing atherosclerotic plaques, and is linked to obesity, insulin resistance, and type 2 diabetes. Fatty tissue releases adipocytokines that cause insulin resistance, endothelial dysfunction, hypercoagulability, and systemic inflammation to accelerate the atherosclerotic process. Inflammatory adipocytokines (e.g., TNF- $\alpha$, IL-6, MCP-1, leptin, and resistin) increase to higher levels in visceral obesity. In addition, an elevated level of C-reactive protein is associated with an increased risk of myocardial infarction, peripheral artery disease, and diabetes mellitus. ${ }^{28,29}$ Incidentally, a clinical trial undertaken in obese women has indicated that the reduction in body weight attained by dietary changes decreases the level of inflammatory biomarkers and insulin resistance. Adiponectin, an anti-inflammatory and insulin- 
sensitizing adipocytokine, is released as part of the process..$^{30}$ It is important to consider the relationship between the inflammatory process and atherosclerosis and the accelerating role of obesity.

\section{Obesity and Dyslipidaemia}

Lipid disorders found in obese patients include elevated triglycerides, VLDL, Apo B, and non-HDL cholesterol levels, both of which are typically found. The levels of HDL cholesterol and Apo A-I are typically low. LDL cholesterol levels are commonly found in the normal range, but increase in small dense LDLs are often seen. ${ }^{31,32}$ These small, dense LDL particles are thought to be more pro-atherogenic than large LDL particles for a variety of reasons. They reduce affinity to the LDL receptor resulting in a prolonged period in circulation and these small particles penetrate the arterial wall more quickly than large particles and then bind more avidly to intra-arterial proteoglycans, which trap them in the arterial wall. Finally, thin, compact LDL particles are more vulnerable to oxidation, which might lead to increased absorption of macrophages. Postprandial triglyceride levels are also raised in obese subjects and these chylomicron remains are pro-atherogenic. The higher they rise in $\mathrm{BMI}$, the higher the defects in lipid levels. About 60-70 percent of patients who are obese have dyslipidemia, while 50-60 percent of patients who are overweight have dyslipidemia. ${ }^{31}$ Obesity in children and young adults also contributes to increased incidence of elevated triglycerides and reduced HDL cholesterol levels. ${ }^{33}$ The increased risk of cardiovascular disease in obese patients is largely due to this dyslipidemia. ${ }^{34}$

\section{Obesity and Cerebrovascular Disease}

CD refers to a constellation of events that trigger interruptions in the blood flow to the brain that result in a lack of brain activity. The brain is a highly specialized and incredibly responsive organ, it requires enough blood supply because it has insufficient energy capacity. ${ }^{35}$ Every year about 15 million individuals globally encounter some form of the CD along with significant neurological deficits. ${ }^{36}$ However, in the present year, the $\mathrm{CD}$ has been identified as the second most prevalent cause of death following ischemic heart disease (IHD) and the leading cause of disability. The most frequent type of $\mathrm{CD}$ is a temporary ischemic attack, accounting for around 40 percent of the total cases. Other forms such as cerebral embolism (30 \%) and cerebral thrombosis (20\%) cover rest of the percentage. ${ }^{37}$

\section{Obesity and Gastrointestinal Abnormalities}

The gastrointestinal tract plays a key role in obesity by leading to saturation and satiety, generating intestinal hormones that control appetite (such as ghrelin, cholecystokinin, and peptide YY), incretins (such as glucagonlike peptide-1) that affect postprandial glycaemia, absorption of nutrients that eventually decide the positive energy balance that results in obesity, Changes in bile acids and microbiomes and metabolic products of microbial nutrient digestion (short-chain fatty acids) that enhance some of the metabolic factors associated with obesity. ${ }^{38}$

\section{Obesity and Cancer}

Evidence is growing about the potential impact of sex hormones and the connection between obesity and cancer, primarily cancers known to be hormone-dependent, such as endometrial, breast, uterine, ovarian and prostate cancers. ${ }^{39}$ The sex steroid hormones are primarily developed by the adrenal glands, and the endogenous hormones include estrogens (E1-estrone and E2-estradiol), androgens (testosterone, androstenedione, dihydrotestosterone, and dehydroepiandrosterone) and progestogens (such as progesterone). ${ }^{40}$ For example, estrogen binds to the receptor (ER), triggering intracellular signalling pathways that will initiate tumour progression by stimulating the cell division. In addition, excess aromatase from the adipose tissue may contribute to even higher levels of estradiol, which are not expected to cause further damage to DNA. Estrogens also interfere with IGF, which stimulates tumour growth through the inhibition of apoptosis. Adipokines, hormones derived by adipose tissue, have also been identified as a potential correlation between obesity and cancer. Leptin is the most well-known adipokine; however, adiponectin, resistin and visfatin have also been studied. ${ }^{39}$ Leptin hormone indirectly suppresses hunger by elevated levels of melanocytestimulating hormone and binding of melanocyte receptors. 40 Colon, prostate, and breast cancers have been identified with elevated serum leptin levels. However, the accuracy of the influence of leptin on tumorigenesis in different forms of cancer is still being investigated. Adiponectin is negatively associated with cancer risk in endometrial, breast, colon, and prostate cancers. The influence of resistin and visfatin was not well studied. To date, resistance has only been examined in small samples. Possible mechanisms for tolerance may be linked to inflammatory and angiogenic pathways. ${ }^{40}$ Visfatin is predominantly studied in vitro and patients with type II diabetes because it mimics the action of insulin. To date, the association between elevated levels of visfatin and cancer has only been identified in patients with colorectal cancer. There is substantial evidence of the association between obesity and certain cancers. These include gallbladder cancer, oesophagus (adenocarcinoma), thyroid cancer, kidney cancer, prostate cancer, bowel cancer and breast cancer. ${ }^{41}$ This correlation has been further reinforced by the fact that there is a decreased prevalence of cancer and weight loss mortality. ${ }^{42}$ However, the underlying reason for relating these cancers to obesity and uterus and breast cancers are believed to be linked to elevated levels of estrogen synthesized from fat tissue in obese individuals. ${ }^{43}$

\section{Obesity and Reproductive Disease}

Polycystic ovarian syndrome (PCOS), marked by anovulation, hyperandrogenism and polycystic ovaries, is linked with both obesity and insulin resistance. It has been reported that elevated visceral fat estimated by waist circumference of more than $88 \mathrm{~cm}$ is correlated with hyperandrogenemia in patients with PCOS and that reducing insulin tolerance by weight reduction or through medications that raise peripheral insulin sensitivity contributes to enhanced hormonal aberrations and ovulation. ${ }^{4}$

In males, abdominal obesity has been associated with impotence and infertility. In a single-blinded randomized controlled study of 110 obese men with erectile disorders but 
with no other risk factors, such as diabetes, hyperlipidemia, hypertension, the sexual performance associated with reduced BMI was improved. There are other reproductive risks with obesity that arise during pregnancy and work. These include gestational diabetes, macrosomia, dystocia, and elevated frequency of caesarean section. ${ }^{45}$

Obesity has a negative effect on reproductive ability, mainly due to the functional modification of the hypothalamic-pituitary-ovary (HPO) axis. Obese women also have elevated serum insulin levels, which is a recognized trigger for increased development of ovarian androgen.

These androgens are aromatized to estrogen at elevated concentrations in the periphery due to excess adipose tissue, leading to negative feedback on the HPO axis and influencing the development of gonadotropin. This is expressed as menstrual abnormalities and ovulatory failure. Hyperinsulinemia is strongly involved in polycystic ovarian syndrome (PCOS) pathogenesis, characterized by oligomenorrhea and hyperandrogenism. Obesity leads to insulin resistance which tends to intensify the effects of PCOS, with obese women frequently displaying a more extreme phenotype.

Elevated androgen levels in PCOS lead to the accumulation of visceral fat, contributing to insulin resistance with hyperinsulinemia, further increasing the development of the ovary and adrenal androgens in a constant loop. The prevalence of PCOS in some obese populations is close to 30 $\%$, but the causative role of obesity in the production of PCOS has not been identified". 46

\section{Obesity and Psychosocial Problems}

Several processes can mediate the connection between early life trauma and overweight. That includes higher dietary skipping of meals for weight loss, unhealthy eating-related problems and habits, increased use of food in response to stress, and decreased physical activity. In addition, elevated levels of depression and hypothalamic-pituitary-adrenal axis dysregulation, both known to raise the risk of overweight/obesity, were observed in girls with a history of childhood abuse relative to controls. Interestingly, the severity of early childhood trauma is a strong indicator of subsequent overweight/obesity, particularly after adjusting for minority status and disability-related medical comorbidity. ${ }^{47-49}$

\section{CONCLUSIONS}

The present review concludes that obesity leads to different disease conditions, and the best way to treat obesity is to maintain a healthy weight, dietary changes by reducing calories intake, do moderate physical activity to prevent further weight gain or maintain the loss of a modest amount of weight.

Financial or other competing interests: None.

Disclosure forms provided by the authors are available with the full text of this article at jemds.com.

\section{REFERENCES}

[1] Zimmet P. Globalization, coca-colonization and the chronic disease epidemic: can the Doomsday scenario be averted? J Intern Med 2000;247(3):301-10.

[2] Butola ML, Ambad RS, Butola ML, et al. Clinical correlation of oxidative stress andantioxidant in obese individuals. European Journal of Molecular \& Clinical Medicine 2021;8(1):349-55.

[3] Sirtori A, Brunani A, Villa V, et al. Obesity is a marker of reduction in QoL and disability. Scientific World Journal 2012;2012:167520.

[4] Lata Kanyal MT, Mujawar A. Status of vitamin b12 in type 2 diabetes mellitus patients taking metformin based oral hypoglycemic agent-a cross sectional study. Indian Journal of Basic and Applied Medical Research 2019;1(9):18-26.

[5] Madhura TK, Kanyal L, Mujawar A. Effect of glycemic control on vitamin b12 status in type 2 diabetes mellitus. Indian Journal of Basic and Applied Medical Research 2019;1(9):7-17.

[6] Butola LK, Meshram A, Dhok A, et al. Urticaria due to adverse drug reaction in diabetes: a case presentation. J Evolution Med Dent Sci 2020;9(37):2764-6.

[7] Røder ME, Porte D, Schwartz RS, et al. Disproportionately elevated proinsulin levels reflect the degree of impaired B cell secretory capacity in patients with noninsulin-dependent diabetes mellitus. J Clin Endocrinol Metab 1998;83(2):604-8.

[8] Ambad R, Jha RK, Butola LK, et al. Relationship between uric acid and creatinine in pre-diabetic and diabetic patients: Vidarbha region of Maharashtra. Int J Res Pharm Sci 2020;11(3):3412-7.

[9] Karpe F, Dickmann JR, Frayn KN. Fatty acids, obesity, and insulin resistance: time for a reevaluation. Diabetes 2011;60(10):2441-9.

[10] Butola LK, Gusain N. To study the association of vitamin D, calcium and phosphorus in type 2 diabetes mellitus patients. Int J Recent Sci Res 2020;11(10):39749-52.

[11] Jha RK, Kondhalkar A, Jain P, et al. Supplementation of vitamin $\mathrm{D}$ in diabetic patients. Indian Journal of Forensic Medicine Toxicology 2020;14(4):7016-7.

[12] Jiang SZ, Lu W, Zong XF, et al. Obesity and hypertension. Exp Ther Med 2016;12(4):2395-9.

[13] Coelho M, Oliveira T, Fernandes R. Biochemistry of adipose tissue: an endocrine organ. Arch Med Sci 2013;9(2):191-200.

[14] De Mey JGR, Vanhoutte PM. End of the line revised: moving on from nitric oxide to CGRP. Life Sci 2014;118(2):120-8.

[15] Tucker PS, Dalbo VJ, Han T, et al. Clinical and research markers of oxidative stress in chronic kidney disease. Biomarkers 2013;18(2):103-15.

[16] Ashburn DD, DeAntonio A, Reed MJ. Pulmonary system and obesity. Crit Care Clin 2010;26(4):597-602.

[17] Laghi F, Tobin MJ. Disorders of the respiratory muscles. Am J Respir Crit Care Med 2008;168(1):10-48.

[18] Global initiative for chronic obstructive lung disease. Global Strategy for the diagnosis, management, and prevention of chronic obstructive pulmonary disease. Executive Summary. [Accessed 2020 Sep,24]. Available from: 
http://www.goldcopd.org/Guidelineitem.asp?l1=2\&l2=1 \&intId=2180.

[19] Isono S. Obstructive sleep apnea of obese adults: pathophysiology and perioperative airway management. Anesthesiology 2009;110(4):908-921.

[20] Leone N, Courbon D, Thomas F, et al. Lung function impairment and metabolic syndrome: the critical role of abdominal obesity. Am J Respir Crit Care Med 2009;179(6):509-16.

[21] Ouchi N, Parker JL, Lugus JJ, et al. Adipokines in inflammation and metabolic disease. Nat Rev Immunol 2011;11(2):85-97.

[22] Gomez R, Conde J, Scotece M, et al. What's new in our understanding of the role of adipokines in rheumatic diseases? Nat Rev Rheumatol 2011;7(9):528-36.

[23] Otero M, Lago R, Lago F, et al. Signalling pathway involved in nitric oxide synthase type II activation in chondrocytes: synergistic effect of leptin with interleukin-1. Arthritis Res Ther 2005;7(3):R581-91.

[24] de Boer TN, van Spil WE, Huisman AM, et al. Serum adipokines in osteoarthritis; comparison with controls and relationship with local parameters of synovial inflammation and cartilage damage. Osteoarthritis Cartilage 2012;20(8):846-53.

[25] Andriacchi TP, Mundermann A. The role of ambulatory mechanics in the initiation and progression of knee osteoarthritis. Curr Opin Rheumatol 2006;18(5):514-8.

[26] Bennell KL, Hunt MA, Wrigley TV, et al. Role of muscle in the genesis and management of knee osteoarthritis. Rheum Dis Clin North Am 2008;34(3):731-54.

[27] Roubenoff R. Sarcopenic obesity: does muscle loss cause fat gain? Lessons from rheumatoid arthritis and osteoarthritis. Ann N Y Acad Sci 2000;904:553-7.

[28] Shoelson SE, Herrero L, Naaz A. Obesity, inflammation, and insulin resistance. Gastroenterology 2007;132(6):2169-80.

[29] Pradhan AD, Manson JE, Rifai N, et al. C-reactive protein, interleukin 6, and risk of developing type 2 diabetes mellitus. JAMA 2001;286(3):327-34.

[30] Esposito K, Pontillo A, Di Palo C, et al. Effect of weight loss and lifestyle changes on vascular inflammatory markers in obese women: a randomized trial. JAMA 2003;289(14):1799-804.

[31] Bays HE, Toth PP, Kris-Etherton PM, et al. Obesity, adiposity, and dyslipidemia: a consensus statement from the National Lipid Association. J Clin Lipidol 2013;7(4):304-83.

[32] Xiao C, Dash S, Morgantini C, et al. Pharmacological targeting of the atherogenic dyslipidemia complex: the next frontier in CVD prevention beyond lowering LDL cholesterol. Diabetes 2016;65(7):1767-78.

[33] Klop B, Elte JW, Cabezas MC. Dyslipidemia in obesity: mechanisms and potential targets. Nutrients 2013;5(4):1218-40.
[34] Skinner AC, Perrin EM, Moss LA, et al. Cardiometabolic risks and severity of obesity in children and young adults. N Engl J Med 2015;373(14):1307-17.

[35] Miller AA, Budzyn K, Sobey CG. Vascular dysfunction in cerebrovascular disease: mechanisms and therapeutic intervention. Clin Sci (London) 2010;119(1):1-17.

[36] World-Heart-Federation. Stroke. http://www.worldheartfederation.org/cardiovascular-health/stroke.

[37] Liu J, Liao X, Wang N, et al. Serum miR-124 and TNF-a are Biomarkers of Ischemic Cerebrovascular Disease. Proceedings of the 2012 International Conference on Applied Biotechnology 2012.

[38] Camilleri M, Malhi H, Acosta A. Gastrointestinal complications of obesity. Gastroenterology 2017;152(7):1656-70.

[39] Roberts DL, Dive C, Renehan AG. Biological mechanisms linking obesity and cancer risk: new perspectives. Annu Rev Med 2010;61:301-16.

[40] Nock NL, Berger NA. Obesity and cancer: overview of mechanisms. Cancer and energy balance, epidemiology and overview. New York, NY: Springer 2010:129-79.

[41] Renehan AG, Tyson M, Egger M, et al. Body mass index and the incidence of cancer: a systematic review and meta-analysis of prospective observational studies. lancet 2008;371(9612):569-78.

[42] Adams TD, Stroup AM, Gress RE, et al. Cancer incidence and mortality after gastric bypass surgery. Obesity (Silver Spring) 2009;17(4):796-802.

[43] Schmandt RE, Iglesias DA, Co NN, et al. Understanding obesity and endometrial cancer risk: opportunities for prevention. Am J Obstet Gynecol 2011;205(6):518-25.

[44] Ehrmann DA. Polycystic ovary syndrome. N Engl J Med 2005;352(12):1223-36.

[45] Dietl J. Maternal obesity and complications during pregnancy. J Perinat Med 2005;33(2):100-5.

[46] Alvarez-Blasco F, Botella-Carretero JI, San Millan JL, et al. Prevalence and characteristics of the polycystic ovary syndrome in overweight and obese women. Arch Intern Med 2006;166(19):2081-2086.

[47] Butola LK, Kanyal D, Ambad R, et al. Role of omega 3 fatty acids, vitamin D, vitamin B12, vitamin B6 and folate in mental wellbeing-a short review of literature. Indian Journal of Forensic Medicine \& Toxicology 2021;15(2):283-8.

[48] Ambad RS, Butola LK, Singh BR, et al. A cross-sectional comparison of minerals in psychiatric disorder. International Journal of Psychosocial Rehabilitation 2020;24(6):5968-76.

[49] D’Argenio A, Mazzi C, Pecchioli L, et al. Early trauma and adult obesity: is psychological dysfunction the mediating mechanism? Physiol Behav 2009;98(5):543-6. 